\title{
Microstructure based model for sound absorption predictions of perforated closed-cell metallic foams
}

\author{
Fabien Chevillotte ${ }^{a)}$ \\ GAUS, Department of Mechanical Engineering, Université de Sherbrooke, Quebec J1K 2R1, Canada \\ Camille Perrot \\ Laboratoire Modélisation et Simulation Multi Echelle (MSME), UMR 8208 CNRS, Université Paris-Est, \\ 5 Boulevard Descartes, 77454 Marne-la-Vallée, France \\ Raymond Panneton \\ GAUS, Department of Mechanical Engineering, Université de Sherbrooke, Quebec J1K 2R1, Canada
}

(Received 12 October 2009; revised 24 June 2010; accepted 7 July 2010)

\begin{abstract}
Closed-cell metallic foams are known for their rigidity, lightness, thermal conductivity as well as their low production cost compared to open-cell metallic foams. However, they are also poor sound absorbers. Similarly to a rigid solid, a method to enhance their sound absorption is to perforate them. This method has shown good preliminary results but has not yet been analyzed from a microstructure point of view. The objective of this work is to better understand how perforations interact with closed-cell foam microstructure and how it modifies the sound absorption of the foam. A simple two-dimensional microstructural model of the perforated closed-cell metallic foam is presented and numerically solved. A rough three-dimensional conversion of the two-dimensional results is proposed. The results obtained with the calculation method show that the perforated closed-cell foam behaves similarly to a perforated solid; however, its sound absorption is modulated by the foam microstructure, and most particularly by the diameters of both perforation and pore. A comparison with measurements demonstrates that the proposed calculation method yields realistic trends. Some design guides are also proposed.
\end{abstract}

(C) 2010 Acoustical Society of America. [DOI: 10.1121/1.3473696]

PACS number(s): 43.55.Ev, 43.20.Gp, 43.30.Ky [LMW]

Pages: $1766-1776$

\section{INTRODUCTION}

How do local geometry parameters relate to sound absorption spectrum in perforated closed-cell metallic foams and how the latter compare to perforated rigid solids? This question arises with the continuously increasing interest to metallic foams combined attractive properties. ${ }^{1}$ Recently, the study of sound absorption enhancement of closed-cell metallic foams, especially its relation to the microstructure of a porous medium, was the focus of much attention. Among the various results of these studies, it was found that (1) techniques based on either rolling or hole drilling are resulting in sound absorption enhancement, ${ }^{2,3}$ and (2) progress can be made in the understanding of their normal sound absorption properties in relation with their local geometry parameters. ${ }^{4}$ The purpose of this work is to carry out a trend analysis to clarify the microphysical foundations of the sound absorption of perforated closed-cell metallic foams and how it compares to perforated rigid solids.

Open and closed-cell metallic foams are used for structural, thermal and acoustic applications. An extensive literature exists for open-cell materials. Incidentally, the most used acoustic model is the one proposed by Biot $^{5,6}$ in which the porous medium is modeled as two superimposed phases (fluid and solid). The fluid phase (usually air) forms an in-

\footnotetext{
a) Author to whom correspondence should be addressed. Electronic mail: fabien.chevillotte@2006.ecam.fr
}

terconnected cell network that opens to the surrounding medium. Considering the skeleton as being rigid, the energy carried by the waves is mainly dissipated through viscous losses (due to relative motion between the two phases), and thermal losses. ${ }^{6}$ Contrary to open-cell foams, closed-cell foams are poor sound absorbers. However, they generally present a better structural rigidity and a lower production cost than open-cell foams.

Two main methods can be used for enhancing the sound absorption of a closed-cell foam. The first method consists in fracturing its cell walls via compression or rolling. ${ }^{2,3}$ In an effort to model the acoustic properties of such a porous medium, it was shown that the fractured foam may be seen as a semiopen-cell material. ${ }^{4}$ The second method consists in hole drilling the closed-cell foam. ${ }^{2}$ Those two methods follow the same principle, which aims at increasing the viscous effects by enabling a relative motion between the two phases of the porous medium. The existing literature on both methods revealed good practical results; however it is limited in the sense that it does not systematically quantify the effects of microstructure modifications introduced by compression, rolling or hole drilling.

In an attempt to offer optimized sound absorbing cellular structures, Wang and Lu considered regular and random distributions of cell sizes made of uniform tubes of arbitrary cross section shapes ${ }^{7}$ for which semi-analytic expressions exist. An interesting conclusion is that well chosen cross section size and shape can significantly increase the sound ab- 
sorption performances. For more complicated geometries, microphysical insight is given from first-principle calculations through the application of the homogenization procedure. ${ }^{8}$ Furthermore, some other works have demonstrated the potential of simple two-dimensional models of microstructures in order to analyze the acoustical macrobehavior of real open-cell foams. ${ }^{9,10}$ Note that, in the context of sound absorption in metallic foams, such a microstructural approach, based on periodic unit cells (PUC), could be applied to analyze the effect of techniques relying on either rolling or hole drilling. Similar trends between semiopen-cell and open-cell materials are anticipated, because the crack size in semiopen-cell materials is analog to the throat size in open-cell materials. In both cases, this is actually the smallest aperture in which the long-wavelength wave can penetrate in the porous media.

In this paper, the focus is on the long-wavelength acoustic behavior of perforated closed-cell metallic foams. Thus, a microstructural model which is not limited to uniform tubes of arbitrary cross section shapes, but rather based on an array of spherical pores connected by perforations has to be developed. This work extends the PUC microstructural approach to better understand and quantify the effects of perforations in closed-cell metallic foams on the enhancement of sound absorption. In particular, a parametric study will focus on five local geometry descriptors: the perforation diameter $D_{\text {hole }}$, the perforation density $n$, the distance between spherical pores $d$, the relative position between spherical pores and perforations $w$, and the pore diameter $a$.

Section II of this paper presents the theoretical background behind the developments. First, a robust equivalentfluid model ${ }^{11}$ is recalled. This model enables predicting the long-wavelength acoustic absorption of semiopen-cell and open-cell porous media on the basis of five asymptotic macroscopic parameters. Then, a $2 \mathrm{D}$ microstructural model is proposed to represent the typical local geometry of a perforated closed-cell metallic foam. The numerical solutions of the boundary value problems governing the physics in the presented 2D model are provided in order to compute its five macroscopic parameters. Also, 2D/3D correction factors are provided to convert the found 2D macroscopic parameters into approximate evaluations of the corresponding 3D macroscopic parameters. Section III presents a detailed study of the effects of the local-geometry parameters on the longwavelength acoustical absorption of a perforated closed-cell metallic foam. Finally, the suggested microstructural approach is used to predict the normal incidence sound absorption of a real perforated aluminum closed-cell foam tested in a standing wave tube.

\section{THEORETICAL BACKGROUND}

\section{A. Acoustical modeling of rigid porous media \\ 1. Equivalent fluid model for open-cell media}

The macroscopic description of long-wavelength acoustic propagation and dissipation phenomena through an interconnected fluid filled rigid porous media may be modeled as an equivalent fluid $^{11}$ characterized by two complex frequency-dependent functions: the dynamic density $\rho_{e q}(\omega)$ which takes into account the visco-inertial interaction between the frame and the saturating fluid, and the dynamic bulk modulus $K_{e q}(\omega)$, which takes into account the thermal interaction. These dynamic functions can be respectively derived from macroscopic parameters using different semiphenomenological models. ${ }^{11-16}$

Johnson et al. ${ }^{12}$ used four macroscopic parameters for modeling the visco-inertial interaction: the open porosity $\phi$, the static airflow resistivity $\sigma$, the tortuosity $\alpha_{\infty}$, and their newly introduced viscous characteristic length $\Lambda$. Similarly, Champoux and Allard ${ }^{13}$ introduced one additional macroscopic parameter for modeling the thermal interaction: the thermal characteristic length $\Lambda^{\prime}$. Note that the way the problem is addressed can eventually be refined ${ }^{14-16}$ by considering additional macroscopic parameters such as the static viscous tortuosity $\alpha_{0}$, the static thermal permeability $k_{0}{ }^{\prime}$, and the static thermal tortuosity $\alpha_{0}{ }^{\prime}$ as successive improvements of the modeled frequency-dependent viscous and thermal dynamic functions.

In this work, for trend investigation purposes, the descriptions of the visco-inertial and thermal interactions between the rigid frame and saturating fluid are restricted to the Johnson et al. ${ }^{12}$ model

$$
\rho_{e q}(\omega)=\frac{\rho_{0} \alpha_{\infty}}{\phi}\left[1-i \frac{\sigma \phi}{\omega \rho_{0} \alpha_{\infty}} \sqrt{1+i \omega \rho_{0} \eta\left(\frac{2 \alpha_{\infty}}{\sigma \phi \Lambda}\right)^{2}}\right]
$$

and the Champoux and Allard ${ }^{13}$ model

$$
\begin{aligned}
\frac{1}{K_{e q}(\omega)}= & \frac{\phi}{\gamma P_{0}}\left\{\gamma-(\gamma-1)\left[1-i \frac{8 \eta}{\omega \rho_{0} \operatorname{Pr} \Lambda^{\prime 2}}\right.\right. \\
& \left.\left.\times \sqrt{1+\frac{i \omega \rho_{0} \operatorname{Pr}}{\eta}\left(\frac{\Lambda^{\prime}}{4}\right)^{2}}\right]^{-1}\right\},
\end{aligned}
$$

where $\omega$ is the angular frequency, $\eta$ is the fluid viscosity, $\rho_{0}$ is the fluid density, $\gamma$ is the specific heat ratio, $P_{0}$ is the atmospheric pressure, and $\operatorname{Pr}$ is the Prandtl number.

Thus, the acoustical macro-behavior description requires five macroscopic parameters for which analytical expressions exist for simple geometries (ex.: cylindrical pores, see $\S$ II.A.2). For more complicated geometries (ex.: open-cell foams), one can use experimental methods or, with a view to carry out a sensitivity analysis of the local geometry parameters, numerical methods. In the following, the finite element analysis is used for solving the asymptotic low (steady Stokes) and high (electric) frequency viscous boundary value problems at the local scale. Then, the macroscopic parameters can be extracted from the fields solving these two asymptotic behaviors as it will be shown in $\S$ II.B.2.

\section{Macroscopic parameters for perforated solids}

For the case of a circular cylindrical pore, $\phi=1, \alpha_{\infty}$ $=1, \Lambda=\Lambda^{\prime}=R_{\text {hole }}, \sigma=8 \eta / \Lambda^{2}$ where $R_{\text {hole }}$ is the radius of the cylinder. From there, one can derive the macroscopic parameters of a solid containing circular cylindrical perforations. Considering a density of $n$ pores per unit area of surface, the macroscopic parameters of the perforated solid are

$$
\phi=n \pi R_{\text {hole }}^{2},
$$




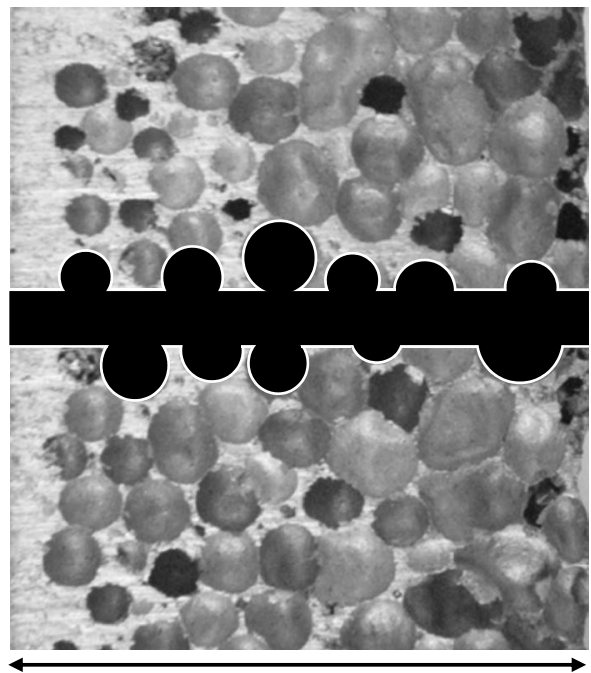

$26.4 \mathrm{~mm}$

FIG. 1. Example of a perforation through a real closed-cell aluminum foam sample.

$$
\begin{aligned}
& \sigma=\frac{8 \eta}{n \pi R_{\text {hole }}^{4}}, \\
& \alpha_{\infty}=1, \\
& \Lambda=\Lambda^{\prime}=R_{\text {hole }} .
\end{aligned}
$$

\section{Sound absorption evaluation}

Assuming an absorbing porous layer of thickness $L_{s}$ that is backed by a rigid wall, under normal incidence acoustic plane wave, the sound absorption coefficient is

$$
\alpha=1-\left|\frac{Z_{n}-1}{Z_{n}+1}\right|^{2},
$$

with the normalized surface impedance of the porous medium defined as

$$
Z_{n}=\frac{Z_{e q}}{\rho_{0} c_{0}} \operatorname{coth} i k_{e q} L_{s},
$$

where $c_{0}$ is the sound speed in air, and $Z_{e q}$ and $k_{e q}$ are the characteristic impedance and complex wave number of the porous medium. They are given by $Z_{e q}=\sqrt{\rho_{e q} K_{e q}}$ and $k_{e q}$ $=\omega \sqrt{\rho_{e q} / K_{e q}}$.

\section{B. Microstructural model}

\section{Local 2D geometry}

A typical closed-cell aluminum foam is shown in Fig. 1. Such a foam can be modeled as a pack of air-filled spherical pores. Contrary to open-cell foams, this pack does not form an interconnected network of pores opened to the surrounding free-fluid medium. Consequently, an acoustic P2 compressional wave cannot penetrate and propagate in the fluid phase to dissipate its energy through visco-inertial forces (i.e., there is no relative motion between the frame and the

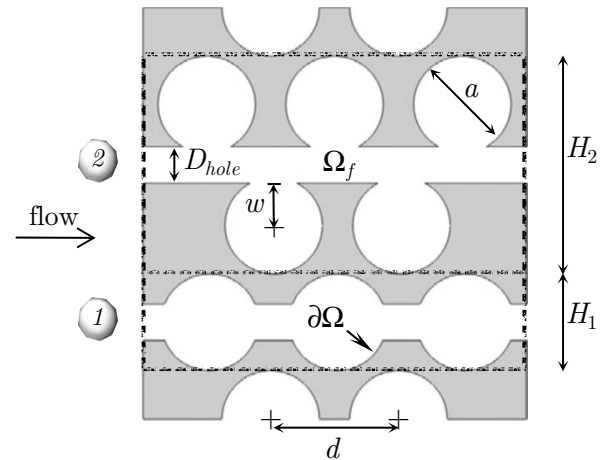

FIG. 2. Two-dimensional model geometry of a perforated closed-cell metallic foam. Two periodic unit cell (PUC) configurations are depicted: (1) lined-up and (2) alternated.

air filling the pores). Such metallic foams are poor sound absorbers and the equivalent fluid model of $\S$ II.A.1 is useless.

With the aim of improving sound absorption of closedcell metallic foams, one needs to promote relative motion between the frame and the saturating fluid by drilling holes through the closed-cell medium. The perforations are opened to the surrounding air and interconnect with some cells as depicted in black for a single perforation in Fig. 1. On this real aluminum foam sample, one easily realizes that the pores may be interconnected differently depending on the position of the perforation. A priori, the way the pores are interconnected through the perforation may influence the velocity field patterns through the network and, as a consequence, the acoustical performances.

To simplify the numerical analysis, a local geometry having a regular repartition of monodispersed pores is first considered. This is shown in Fig. 2. In this model, five characteristic parameters define the local geometry configuration: the diameter of the pores $a$, the diameter of perforations $D_{\text {hole }}$, the distance between pores $d$, the relative position between pores and perforations $w$, and the position of perforations. Here, two typical configurations of perforations are studied: (1) pores lined up with the perforation, and (2) pores alternated along the perforation. For each local configuration, the fluid domain is $\Omega_{f}$, the fluid-solid interface is $\partial \Omega$, and the total periodic homogenization volume (containing both the solid and fluid phases) is shown by the dashed lines. Note that the input surface of each configuration is characterized by a surface porosity given by $h_{s}=D_{\text {hole }} / H_{i}$ with $i=1,2$.

\section{Computational method}

In order to study the influence of the perforations with respect to the size and distribution of the pores at the local scale, the finite element analysis is used. As shown in what follows, two asymptotic viscous boundary value problems need to be solved at the local scale to deduce the five macroscopic parameters used in the equivalent fluid JohnsonChampoux-Allard (JCA) model.

Assuming a locally incompressible saturating fluid, the low-frequency limit $(\omega \rightarrow 0)$ leading parameter (i.e., the 
static airflow resistivity $\sigma$ ) is deduced from the solution of the scaled steady Stokes problem defined by the following set of equations: ${ }^{17}$

$$
\begin{aligned}
& \Delta \mathbf{w}=\nabla \pi-\mathbf{e} \quad \text { in } \Omega_{f}, \\
& \nabla \cdot \mathbf{w}=0 \quad \text { in } \Omega_{f}, \\
& \mathbf{w}=\overrightarrow{0} \quad \text { on } \partial \Omega,
\end{aligned}
$$

where $\nabla$ and $\Delta$ are the local 2D del and Laplacian differential operators, $\mathbf{w}$ is the local scaled static velocity field in square meter $\left(\mathrm{m}^{2}\right), \mathbf{e}=\left[\begin{array}{ll}1 & 0\end{array}\right]^{\mathrm{T}}$ is a unit vector representing a scaled pressure gradient along the $x$-axis, and $\pi$ is the associated scaled scalar pressure. In what follows, the symbol \langle\rangle designates a fluid-phase average. Writing the pressure $p$ in terms of its mean and deviatoric parts, $p=\langle p\rangle+\Pi$ with $\langle\Pi\rangle$ $=0$, the macroscopic pressure gradient is related to e by $\boldsymbol{\nabla}\langle p\rangle=-|\boldsymbol{\nabla}\langle p\rangle| \mathbf{e}$. The small fluctuation $\Pi$ is related to $\pi$ by $\Pi=|\nabla\langle p\rangle| \pi$. Finally, the static velocity field $v$ is related to $\mathbf{w}$ by $\mathbf{v}=|\boldsymbol{\nabla}\langle p\rangle / \eta| \mathbf{w}$. By imposing the no-slip boundary condition at the fluid-solid interface and the periodicity condition on $\pi$ and $\mathbf{w}$ at the inlet/outlet surfaces, the aforementioned periodic problem can be solved on $\Omega_{f}$ using finite element software (ex.: COMSOL Multiphysics, Freefem++) or another numerical scheme. Once the solution is obtained for a given local geometry, and its convergence ensured, the macroscopic static airflow resistivity is computed by averaging the scaled incompressible velocity field,

$$
\sigma=\frac{\eta}{\phi\left\langle w_{x}\right\rangle}\left[\text { in } \mathrm{Ns} / \mathrm{m}^{4}\right]
$$

where $\left\langle w_{x}\right\rangle$ is the averaged scaled static velocity field along the $x$-axis. The open porosity $\phi$ is computed from the ratio of the mesh volume (i.e., the fluid phase volume) to the total homogenization volume.

In the high-frequency limit $(\omega \rightarrow \infty)$, the viscous boundary layer is small compared to the typical pore size and the fluid tends to be perfect (i.e., without viscosity). Considering that the wavelength is large enough compared to the periodic local geometry (i.e., the homogenization volume), the fluid can be considered as being locally incompressible. Under these conditions, the viscous problem is similar to the electric conduction problem defined by the following set of equations: ${ }^{18}$

$$
\begin{aligned}
& \mathbf{E}=-\nabla \varphi+\mathbf{e} \quad \text { in } \Omega_{f}, \\
& \nabla \cdot \mathbf{E}=0 \quad \text { in } \Omega_{f}, \\
& \mathbf{E} \cdot \mathbf{n}=0 \quad \text { on } \quad \partial \Omega,
\end{aligned}
$$

where $\mathbf{E}$ is the dimensionless scaled electric field that solves the corresponding electric conduction problem for a porous medium filled with a conducting fluid and having an insulating solid phase, $\mathbf{n}$ is the unit outward normal, $\mathbf{e}=\left[\begin{array}{ll}1 & 0\end{array}\right]^{\mathrm{T}}$ is the unit vector defined along the $x$-axis, and $\varphi$ is a stationary or $\Omega$-periodic scalar field. Imposing the Neumann boundary conditions on the fluid-solid interface and the periodicity of $\varphi$ on the inlet/outlet surfaces, the aforementioned periodic

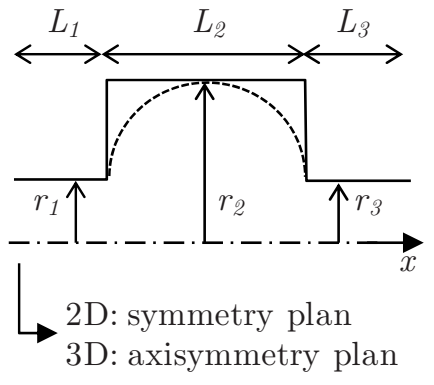

FIG. 3. Basic geometry model for a series of slits (2D) and cylinders (3D).

problem can again be solved on $\Omega_{f}$ using finite element software (ex.: COMSOL Multiphysics, Freefem++) or another numerical scheme. Once the solution is obtained for a given local geometry, and its convergence ensured, one can compute the macroscopic tortuosity from

$$
\alpha_{\infty}=\left\langle E_{x}\right\rangle^{-1},
$$

where $\left\langle E_{x}\right\rangle$ is the averaged scaled electric field along the $x$-axis. The other high-frequency macroscopic parameters $(\Lambda$ and $\Lambda^{\prime}$ ) are computed as follows. The thermal characteristic length $\Lambda^{\prime}$, defined as twice the fluid phase volume to wetsurface ratio, is obtained by the volume to wet surface ratio of the mesh. The viscous characteristic length $\Lambda$ is computed from the electric field as shown by Johnson et al. ${ }^{12}$

$$
\Lambda=2 \frac{\int_{\Omega}|E|^{2} d V}{\int_{\partial \Omega}|E|^{2} d S} .
$$

\section{2D/3D approximate conversion factors}

Due to the boundary conditions applied on the geometry, the 2D-microstructural geometry shown in Fig. 2 could be approximated by a series of slits. In the case of a perforated closed-cell metallic foam, the perforation is cylindrical and the pores are spherical. Consequently, axisymmetry seems more appropriate to represent the idealized microgeometry even though it is not exact. Thus, simple analytical relationships are sought for the sake of result representativeness.

Slits or cylinders have been studied for a long time. Champoux and Stinson have studied a series of pores with different shape factors. ${ }^{19,20}$ These works enable to analytically obtain the macroscopic parameters for a series of simple tubes. Figure 3 shows how to approximate the microstructural model of Fig. 2 with a series of 3 slits (in 2D) or 3 circular cylinders (in 3D). This model is called elementary cell. This elementary cell has a total length $L_{T}$ and three sections described by half elementary height or radius $r_{i}$ and length $L_{i}$, where $i=1,2$, or 3. As it will be shown in Sec. III C, the relative position between pores and perforations does not have a significant impact on the sound absorption. Consequently, the elementary cell shown in Fig. 3, where the pore is lined up with the perforation, appears to be convenient enough for developing the approximated 2D/3D relations. 
TABLE I. Analytical expressions of the macroscopic parameters for a series of elementary slits and cylinders with their respective ratios providing approximate $2 \mathrm{D} / 3 \mathrm{D}$ conversion factors.

\begin{tabular}{|c|c|c|}
\hline Series of elementary slits & Series of elementary cylinders & Ratios \\
\hline$\phi_{\text {slit }}=\frac{h_{s}}{3}(1+2 \varepsilon)$ & $\phi_{c y l}=\frac{h_{c}}{3}\left(1+2 \varepsilon^{2}\right)$ & $\frac{\phi_{3 \mathrm{D}}}{\phi_{2 \mathrm{D}}} \cong \frac{\phi_{c y l}}{\phi_{\text {slit }}}=\frac{h_{c}}{h_{s}} \frac{1+2 \varepsilon^{2}}{1+2 \varepsilon}$ \\
\hline$\sigma_{\text {slit }}=\frac{4 \eta}{D_{\text {hole }}^{2} h_{s}}\left(1+2 / \varepsilon^{3}\right)$ & $\sigma_{c y l}=\frac{32 \eta}{3 D_{h o l e}^{2} h_{c}}\left(1+2 / \varepsilon^{4}\right)$ & $\frac{\sigma_{3 \mathrm{D}}}{\sigma_{2 \mathrm{D}}} \cong \frac{\sigma_{c y l}}{\sigma_{\text {slit }}}=\frac{8}{3} \frac{h_{s}}{h_{c}} \frac{1+2 / \varepsilon^{4}}{1+2 / \varepsilon^{3}}$ \\
\hline$\alpha_{\infty s l i t}=\frac{1}{9}(1+2 \varepsilon)(1+2 / \varepsilon)$ & $\alpha_{\infty \circ y l}=\frac{1}{9}\left(1+2 \varepsilon^{2}\right)\left(1+2 / \varepsilon^{2}\right)$ & $\frac{\alpha_{\infty 3 \mathrm{D}}}{\alpha_{\infty 2 \mathrm{D}}} \cong \frac{\alpha_{\infty \circ y l}}{\alpha_{\infty \text { slit }}}=\frac{\left(1+2 \varepsilon^{2}\right)\left(1+2 / \varepsilon^{2}\right)}{(1+2 \varepsilon)(1+2 / \varepsilon)}$ \\
\hline$\Lambda_{\text {slit }}=D_{\text {hole }} \frac{(1+2 / \varepsilon)}{\left(1+2 / \varepsilon^{2}\right)}$ & $\Lambda_{c y l}=\frac{D_{\text {hole }}}{2} \frac{\left(1+2 / \varepsilon^{2}\right)}{\left(1+2 / \varepsilon^{3}\right)}$ & $\frac{\Lambda_{3 \mathrm{D}}}{\Lambda_{2 \mathrm{D}}} \cong \frac{\Lambda_{c y l}}{\Lambda_{\text {slit }}}=\frac{1}{2} \frac{\left(\varepsilon^{2}+2\right)^{2}}{(\varepsilon+2)\left(\varepsilon^{3}+2\right)}$ \\
\hline$\Lambda_{\text {slit }}^{\prime}=\frac{D_{\text {hole }}}{3}(1+2 \varepsilon)$ & $\Lambda_{c y l}^{\prime}=\frac{D_{\text {hole }}}{2} \frac{1+2 \varepsilon^{2}}{1+2 \varepsilon}$ & $\frac{\Lambda_{3 \mathrm{D}}^{\prime}}{\Lambda_{2 \mathrm{D}}^{\prime}} \cong \frac{\Lambda_{c y l}^{\prime}}{\Lambda_{\text {slit }}^{\prime}}=\frac{3}{2} \frac{1+2 \varepsilon^{2}}{(1+2 \varepsilon)^{2}}$ \\
\hline
\end{tabular}

Therefore, for the elementary cell shown in Fig. 3, the five macroscopic parameters become ${ }^{20}$

$$
\begin{aligned}
& \phi=n \sum_{i} A_{i} \frac{L_{i}}{L_{T}}, \\
& \sigma=\frac{1}{n} \sum_{i} \frac{\sigma_{i}}{A_{i}} \frac{L_{i}}{L_{T}}, \\
& \alpha_{\infty}=\frac{1}{L_{T}^{2}} \sum_{i} A_{i} L_{i} \cdot \sum_{i} \frac{L_{i}}{A_{i}}=\frac{\phi}{n L_{T}} \sum_{i} \frac{L_{i}}{A_{i}}, \\
& \Lambda=2 \frac{\sum_{i} \frac{L_{i}}{A_{i}}}{\sum_{i} \frac{P_{i}}{A_{i}^{2}}} \\
& \Lambda^{\prime}=2 \frac{\sum_{i} A_{i} L_{i}}{\sum_{i} P_{i}},
\end{aligned}
$$

where $A_{i}, P_{i}$ and $\sigma_{i}$ are respectively the area, wet surface and static resistivity of the $i^{\text {th }}$ elementary section $\left(A_{i}=\pi r_{i}^{2}, P_{i}\right.$ $=2 \pi r_{i} L_{i}$ and $\sigma_{i}=8 \eta / r_{i}^{2}$ for a circular cylinder; $A_{i}=2 r_{i}, P_{i}$ $=2 L_{i}$ and $\sigma_{i}=3 \eta / r_{i}^{2}$ for a slit), and $n$ is the density of elementary cells $\left(n=n_{c}\right.$ for the cylindrical pores $\left[\mathrm{in} \mathrm{m}^{-2}\right] ; n$ $=n_{s}$ for the slits $\left[\right.$ in $\left.\mathrm{m}^{-1}\right]$ ).

Typically, this elementary cell should require 6 local parameters (i.e., radius and length for each section: $\left.r_{1}, r_{2}, r_{3}, L_{1}, L_{2}, L_{3}\right)$. However, some simplifications can be brought. First, since the diameter of the perforation is uniform throughout the elementary cell, one considers that $r_{1}$ $=r_{3}$. Also, $r_{1}$ and $r_{2}$ are related to the perforation diameter and pore diameter as follows:

$$
r_{1}=\frac{D_{\text {hole }}}{2} \text { and } r_{2}=\frac{D_{\text {hole }}+a}{2} .
$$

Second, as it will be shown in Sec. III C, the ratio between the perforation diameter and the pore diameter may have a strong impact on the sound absorption behavior. To highlight this point, the following ratio is presented

$$
\varepsilon=\frac{r_{2}}{r_{1}}=1+\frac{a}{D_{\text {hole }}} .
$$

Third, preliminary results have revealed that the relative distance between the pores and the perforations is not really significant (Sec. III B). Thus, $L_{1}$ and $L_{3}$ are considered to be equal and expressed as a function of the pore diameter as follows:

$$
L_{2}=a \quad \text { and } L_{1}=L_{3}=\frac{a}{4} .
$$

From the aforementioned constraints, the number of independent local geometry parameters defining the elementary cell configuration is now reduced to $3: D_{\text {hole }}, a$, and $n$ $\left(n_{c}\right.$ or $\left.n_{s}\right)$. Then, the five macroscopic parameters given by Eqs. (18)-(22) can be simplified as summarized in Table I. In these formulas, $h_{s}$ and $h_{c}$ respectively account for the surface porosities of slit (2D) and cylinder (3D) arrangements: $h_{s}$ $=n_{s} D_{\text {hole }}$ and $h_{c}=n_{c} \pi D_{\text {hole }}^{2} / 4$. Note that the surface porosity $h_{c}$ is equal to the surface porosity of the real perforated solid or closed-cell foam sample.

Hence, starting from a real perforated sample, three independent parameters $\left(D_{\text {hole }}, a\right.$, and $\left.h_{c}\right)$ are measured ( $a$ is usually a representative average value). With these parameters, the two-dimensional microstructural model is built (Sec. II B 1). From it, a first evaluation of the macroscopic parameters is numerically obtained (Sec. II B 2). Using the 2D/3D conversion factors developed in Table I, the macroscopic parameters of the real perforated sample are approximated. Finally, these macroscopic parameters are used in the equivalent fluid model (Sec. II A) to predict the sound absorption of the perforated sample.

\section{RESULTS AND DISCUSSION}

Step by step, the influence of the microstructural parameters on the normal incidence sound absorption coefficient is now studied for three different porous configurations backed by a rigid termination. The configurations are illustrated in 


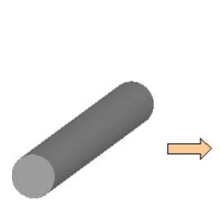

(a)

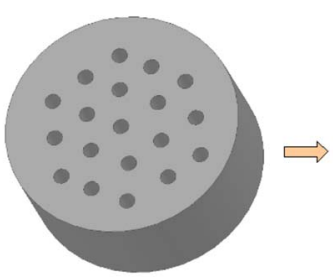

(b)

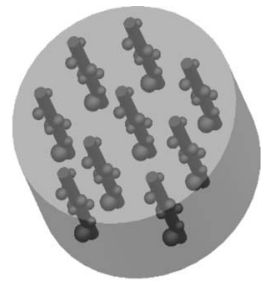

(c)
FIG. 4. (Color online) Main steps of the parametric study: (a) simple uniform tube of circular cross section, (b) perforated solid, and (c) perforated closed-cell metallic foam.

Fig. 4 and the microstructural parameters are those depicted in Fig. 2. First, a single uniform tube of circular cross section is analyzed to point out the existence of a perforation diameter maximizing the normal incidence sound absorption for a given sample length. Second, the case of a solid sample with several perforations of circular cross section is examined to show the influence of the surface porosity or the perforation density. Third, the case of the perforated closed-cell metallic foam is numerically investigated using the $2 \mathrm{D}$ microstructural model introduced in Sec. II B. For this case, the effects of the relative position between the spherical pores and the perforations are first investigated, followed by the effects of the pore size. Finally, the 2D/3D analytical correction factors are used to transpose the $2 \mathrm{D}$ numerical results to $3 \mathrm{D}$ results with a view to compare normal incidence sound absorption predictions to impedance tube measurements.

Note that for all the following simulations, dry air at standard conditions $\left(18{ }^{\circ} \mathrm{C}, 101.3 \mathrm{kPa}\right)$ is considered.

\section{A. Effects of the perforation diameter $\left(\boldsymbol{D}_{\text {hole }}\right)$}

\section{Case of a single uniform tube $(\phi=1)$}

The single uniform tube of circular cross section shown in Fig. 4(a) is considered. The tube is closed at one end and has diameter $D_{\text {hole }}$ and length $L_{s}$. Its macroscopic parameters are analytically obtained as described in Sec. II A 2, and its normal incidence sound absorption computed from Eq. (7).

Figure 5(a) shows the influence of the perforation diameter on the sound absorption coefficient as a function of the dimensionless quantity $k_{0} L_{s}$, where $k_{0}$ is the wave number in air. One can note that the overall sound absorption increases as the diameter increases until it reaches a climax for a specific perforation diameter $D_{\text {spe }}$, and then starts decreasing. Here, $D_{\text {spe }}$ is the diameter for which the sound absorption coefficient is equal to the unity at the first absorption peak (i.e., quarter wavelength absorption at approximately $k_{0} L_{s}$ $=\pi / 2$ ). It is worth mentioning that the diameters used to plot Fig. 5(a) are fractions or multiples of $D_{\text {spe }}$ which depends on length $L_{s}$ as discussed below. Consequently, Fig. 5(a) is a normalized sound absorption graph that fits for all sizes of tubes of circular cross section backed by a rigid termination.

From the analytical expressions given in Sec. II A, the specific perforation diameter $D_{\text {spe }}$ can be expressed for different values of $L_{s}$. The results of the calculations are plotted in Fig. 6 (see $\phi=1$ curve). One can note that a power-law relation exists between $D_{\text {spe }}$ and $L_{s}$, it is given by
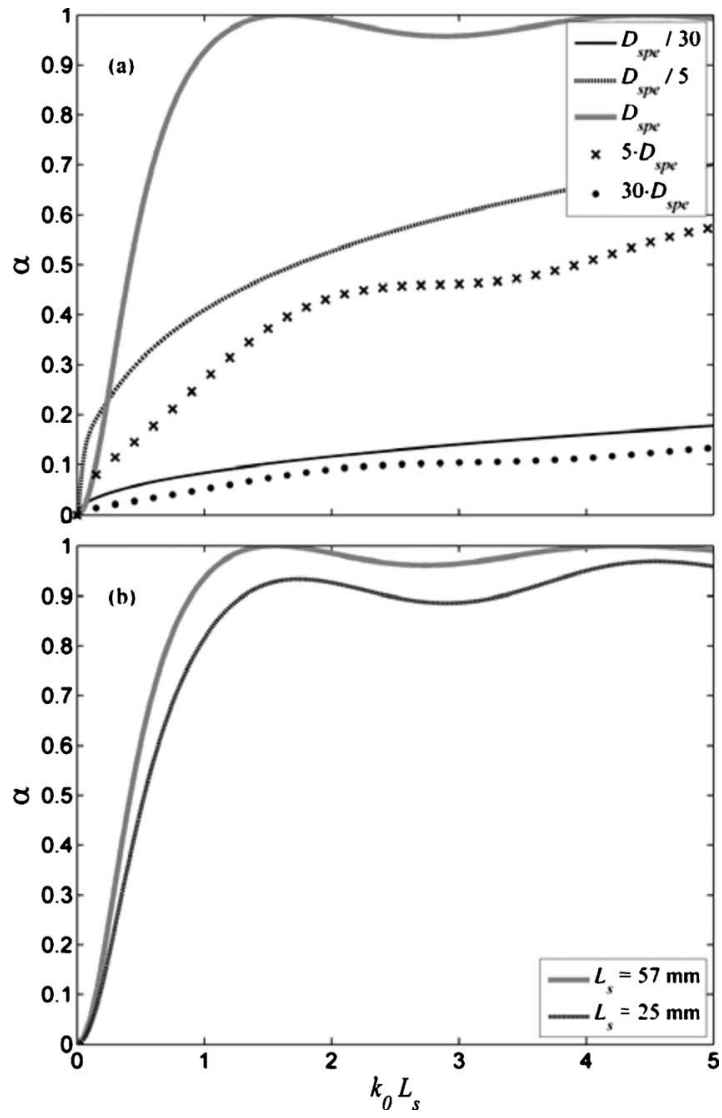

FIG. 5. Sound absorption coefficient of (a) a single cylinder of circular cross section closed at the end and (b) a highly porous melamine foam.

$$
D_{\text {spe }}=\beta \sqrt{L_{s}} \text { or } 2 \Lambda_{\text {spe }}=\beta \sqrt{L_{s}} .
$$

For the case of the studied single uniform tube (i.e., $\phi=1$ ), coefficient $\beta$ is equal to $0.0009228 \mathrm{~m}^{1 / 2}$ and is valid for the used dry air $\left(18{ }^{\circ} \mathrm{C}, 101.3 \mathrm{kPa}\right)$ and a hard back; however, it does not change significantly for typical atmospheric conditions starting from those used in this study. The relation shown in Fig. 6 concurs with results found by Wang and $\mathrm{Lu}^{7}$ and Hakamada et al. ${ }^{21}$ for two specific $D_{\text {hole }}$ to $L_{s}$ ratios. The developed relation generalizes these published results in the sense that it is not limited to specific $D_{\text {hole }}$ to $L_{s}$ ratios.

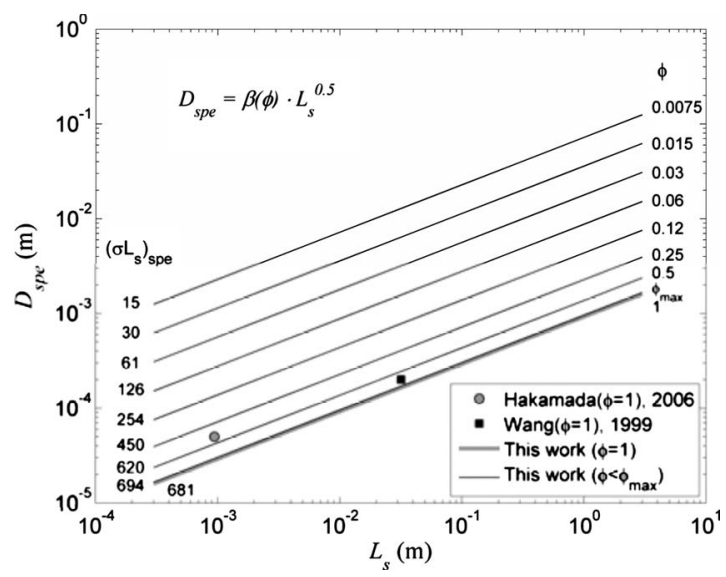

FIG. 6. Power-law relation between specific perforation diameter $D_{\text {spe }}$ and length $L_{s}$ for the perforated solid $\left(\phi \leq \phi_{\max }\right)$. Results for the single tube $(\phi=1)$ are also given. 
The power-law relation of Eq. (26) together with the equations given in Sec. II A 2 for a single tube of circular cross section yields the following relation for the specific resistance

$$
\left(\sigma L_{s}\right)_{s p e}=\frac{32 \eta}{\beta^{2}} \cong 681\left[\text { in } \mathrm{Ns} / \mathrm{m}^{3}\right] .
$$

This relation can be used as a rule of thumb or to understand the sound absorption efficiency of a highly porous sound absorbent. For instance, a melamine open-cell foam has the following macroscopic parameters: ${ }^{22} \quad \phi=0.99, \quad \sigma$ $=12000 \mathrm{Ns} / \mathrm{m}^{4}, \alpha_{\infty}=1.01, \Lambda=100 \mu \mathrm{m}, \Lambda^{\prime}=400 \mu \mathrm{m}$, and $k_{0}^{\prime}=1.5 \times 10^{-9} \mathrm{~m}^{2}$. The last parameter is the thermal permeability in the Lafarge et al. model. ${ }^{15}$ For this foam, porosity and tortuosity are almost equal to 1, like for a single tube. From the aforementioned rule of thumb, the specific term $\left(\sigma L_{s}\right)_{s p e}$ of the melamine foam is reached for a $56.7-\mathrm{mm}$ thickness. This is what is observed in Fig. 5(b). In fact, the sound absorption of the 56.7-mm thick layer of melamine foam is very similar to the one found for the specific tube diameter in Fig. 5(a). If the thickness is lower than $56.7 \mathrm{~mm}$, the overall sound absorption of the foam decreases. Also, using Eq. (26) with a 56.7-mm thickness, one finds that the specific viscous characteristic length is $110 \mu \mathrm{m}$, that is very close to the viscous length of the foam. Note that in this particular case, the predictions presented in Fig. 5(b) used the thermal interaction model worked out by Lafarge et al. ${ }^{15}$ and not the model by Champoux and Allard detailed in Sec. II. Both thermal interaction models are similar for circular tubes; however they may diverge more or less for real sound absorbents.

\section{Case of a perforated solid $(\phi<1)$}

The perforated solid sample shown in Fig. 4(b) is now considered. It has a perforation density $n$ and a thickness $L_{s}$. The cross section of the perforations are circular with diameter $D_{\text {hole }}$. Compared to previous case, this time the porosity may vary from 0 to $\phi_{\max }$ depending on the number of perforations. In reality, the maximum porosity is $\pi / 4$ if the perforations are distributed following a square lattice, or $\pi / 6 / \tan \left(30^{\circ}\right)$ for an hexagonal lattice. Neglecting the nonlinear acoustic response of solid samples with low perforation density, ${ }^{23,24}$ the macroscopic parameters of the perforated solid can be analytically computed from Eqs. (3)-(6), and the normal incidence sound absorption coefficient deduced from Eq. (7).

As shown in Eqs. (3) and (4), the perforation density affects two parameters: porosity $\phi$ and resistivity $\sigma$. In Sec. III A 1, the study of the perforation diameter on a single pore showed that the macroscopic parameter $\sigma$ mainly controls the sound absorption. This was true because the porosity is equal to 1 for a single perforation.

To illustrate the effects of the perforation density or porosity, the sound absorption coefficient of a perforated solid is plotted as a function of $k_{0} L_{s}$ for four different porosities, ${ }^{25}$ see Fig. 7. For each porosity, sound absorption is computed for four different perforation diameters: $D_{\text {spe }} / 30, D_{\text {spe }} / 5$, $D_{s p e}$, and $30 D_{s p e}$, where $D_{s p e}$ is the specific perforation di- ameter yielding $100 \%$ absorption at first peak. The specific resistance $\left(\sigma L_{s}\right)_{s p e}$ corresponding to $D_{s p e}$ is also given for each porosity. It is obtained from Eqs. (3) and (4) assuming $R_{\text {hole }}=D_{\text {spe }} / 2$. Similarly, one can deduce the specific perforation density $n_{\text {spe }}$ corresponding to each porosity from Eq. (3).

In Fig. 7, one can first observe that for a low porosity, the absorption is very selective in terms of frequency and becomes less selective (i.e., bulk absorption) as the porosity increases. A typical selective case is a thin perforated solid with millimetric holes, and a typical bulk absorber case is a thin perforated solid with microperforations (or submillimetric holes). Now, if the curves maximizing the normal sound absorption coefficient for the four porosities are compared (thick lines), one can note that as $\phi$ (or $n$ ) increases, $D_{\text {spe }}$ must be smaller to limit the decrease of $\left(\sigma L_{s}\right)_{\text {spe }}$ and to maintain $100 \%$ absorption at the first peak. Finally, one can observe that the $D_{\text {spe }}$ curve in Fig. 5(a) is very similar to the $D_{\text {spe }}$ curve of the perforated solid relative to $\phi_{\max }$. In this case $\phi_{\max }$ is very close to 1 .

Following the previous discussion, each perforation can be seen as a throat of diameter $D_{\text {hole }}$ by the fluid. If the product $\phi D_{\text {hole }}^{2}$ is too small, the related bulk resistivity is too high. The fluid hardly penetrates the porous medium, acoustic waves are thus reflected and the sound absorption is poor. Inversely, if the product $\phi D_{\text {hole }}^{2}$ is too large, the bulk resistivity is very low. The fluid easily penetrates the porous medium. Thus, little acoustic energy is dissipated by viscous losses and the sound absorption is still poor. In between these two limits, a specific bulk resistivity enables to maximize the sound absorption of the medium.

From the previous analysis of Fig. 7, one can conclude that for a given porosity, there exists a specific perforation diameter $D_{s p e}$. Universal curves to predict $D_{s p e}$ as a function of $L_{s}$ are added in Fig. 6 for $\phi \leq \phi_{\max }$. These curves enable to generalize the power law relation given in Eq. (26) as follows:

$$
D_{\text {spe }}=\beta(\phi) \sqrt{L_{s}}
$$

with, from Eq. (27) for $\phi \neq 1$,

$$
\left(\sigma L_{s}\right)_{s p e}=\frac{32 \eta}{\phi \beta^{2}(\phi)} .
$$

Consequently, Eqs. (28) and (29) together with Fig. 6 can help to quickly design perforated solid absorbers backed by a rigid wall. When considering a perforated solid absorbers backed by a cavity, similar approach can be used. However, the $\beta$ coefficients would be slightly modified. These equations and figures, based on a perforated solid, will also be useful when designing perforated metallic closed-cell foam.

\section{B. Effects of the pore distribution $(w, d)$}

The sound absorption behavior of the perforated closedcell foam sample depicted in Fig. 4(c) is now studied. Once again, the sample is backed by a rigid ending and its thickness is $L_{s}=25 \mathrm{~mm}$. In what follows, three parametric studies are performed to evaluate how the sound absorption is influ- 

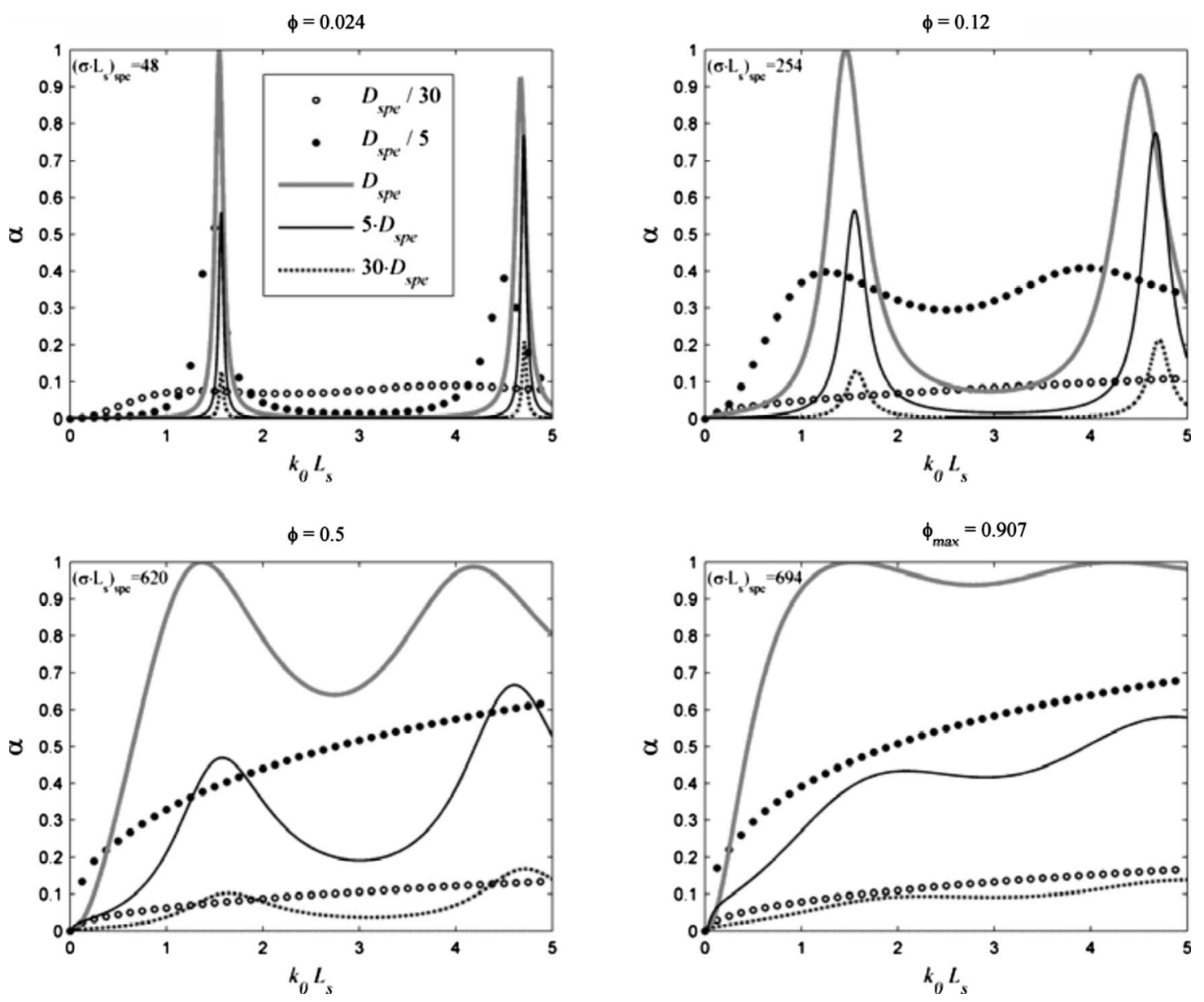

FIG. 7. Sound absorption coefficient of a solid sample with cylindrical perforations of circular cross section closed at the end for four different porosities. The units of airflow resistance $\left(\sigma \cdot L_{s}\right)_{\text {spe }}$ are in $\mathrm{N} \mathrm{s} \mathrm{m}^{-3}$.

enced by (1) the pore alignment, (2) the pore/perforation relative position $w$, and (3) the distance between pores $d$.

For trend investigation purposes, the $3 \mathrm{D}$ configuration is approximated by one of the 2D models proposed in Fig. 2. This time, since no analytical model is available for predicting the sound absorption coefficient of this complex 2D model, the problem is solved following the microstructure based model described in Sec. II B 2. Therefore, one has to fix the perforation configuration (i.e., lined-up or alternated, see Fig. 2), the pore alignment (homogeneous or heterogeneous), the diameter of the perforation $\left(D_{\text {hole }}\right)$, and the three local geometry parameters $(w, d$, and $a)$ described in Fig. 2 . For all the following simulations, unless otherwise indicated, the lined-up configuration is used with $D_{\text {hole }}=1 \mathrm{~mm}$ and $H_{1}=D_{\text {hole }}+a+2 w$.

Once the 2D finite element model is built, its numerical solution yields the corresponding macroscopic parameters (Sec. II B 2). Figure 8 gives an idea of the mesh used for heterogeneous and homogeneous pore distributions. The present work considered quadratic Lagrange elements. These macroscopic parameters are then used with the equivalent fluid model to predict the sound absorption coefficients of the $25-\mathrm{mm}$ thick sample backed on a rigid termination.

To evaluate the effects of the alignment of the pores, two pore distribution patterns are compared: homogeneous and heterogeneous, see Fig. 8. The homogeneous distribution is similar to configuration 1 shown in Fig. 2. For the numerical simulations, $a=1 / 10 D_{\text {hole }}$ and $d=3 / 2 a$. Additionally, for the heterogeneous configuration, the pore/perforation relative position is randomly selected with the constraint that, in average, $\bar{w}=0 \mathrm{~mm} .{ }^{26}$ Figure 9 (a) shows that both configurations yield close results in terms of sound absorption. Also, but not presented here, simulations on the alternated configuration of Fig. 2 have shown that the alternated and line-up configurations yield the same results when $w=0 \mathrm{~mm}$. Consequently, the homogeneous line-up configuration seems sufficient enough to represent the complexity of a heterogeneous pore distribution when $\bar{w}=0 \mathrm{~mm}$.

The influence of $d$ and $w$ are now investigated. In this
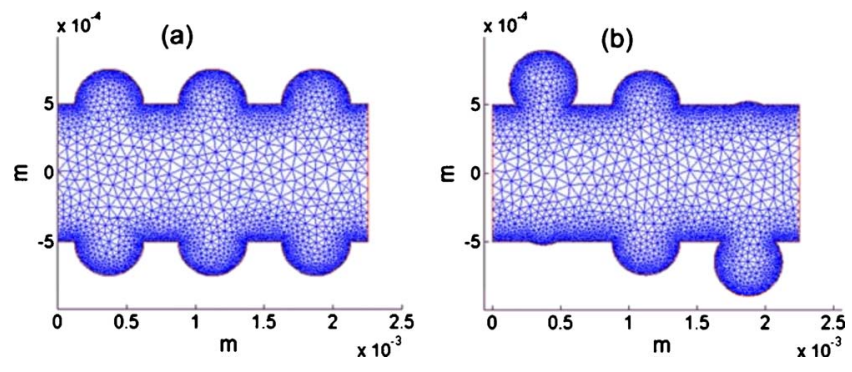

FIG. 8. (Color online) Pore alignment patterns and meshes for the lined-up configuration. (a) Homogeneous distribution pattern with $w=0 \mathrm{~mm}$ and (b) heterogeneous distribution pattern with $\bar{w}=0 \mathrm{~mm}$. 


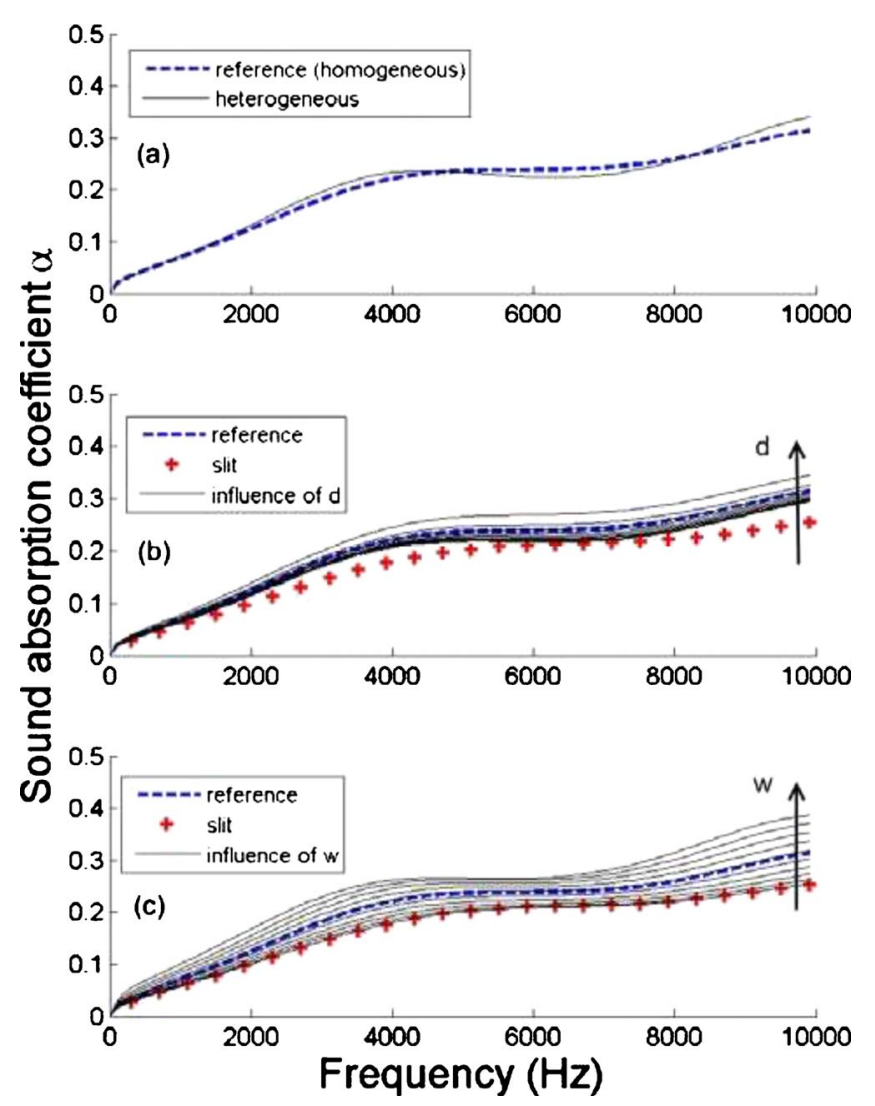

FIG. 9. (Color online) Effects of the pore distribution on the normal sound absorption coefficient. (a) Effects of the pore alignment pattern: homogeneous and heterogeneous. (b) Effects of the distance $d$ between pores. (c) Effects of the relative position $w$ between pores and perforations.

case, $d$ and $w$ are successively varied from the reference homogeneous configuration $\left(D_{\text {hole }}=1 \mathrm{~mm}, a=1 / 10 D_{\text {hole }}\right.$, $d=1.5 a, w=0 \mathrm{~mm})$. Note again that for this reference configuration, simulation results showed that lined-up and alternated configurations yield identical results. The range of variation for $d$ is from $1.05 a$ to $3 a$, and the one for $w$ is from $-0.45 a$ (close to the single slit case of width $D_{\text {hole }}$ ) to $0.45 a$. Following the proposed numerical procedure, the computed sound absorption predictions are presented in Figs. 9(b) and 9(c), respectively. The results are also compared to the sound absorption of a slit and to the one of the reference case. In the used range of variation around the reference configuration, $d$ has few effects on the sound absorption behavior. Similarly, a variation of $w$ has a limited impact on the sound absorption. However, contrary to $d, w$ has lower and upper physical limits. The upper limit is when $w=a / 2$ (i.e., perforation does not hit pores), and its lower limit is $w=-a / 2$ (i.e., pores fall within the perforation)- the lower limit yields identical results to the single slit case.

To conclude, the previous analysis has shown that the local geometry parameters governing the pore/perforation relative position do not have significant effects on the sound absorption behavior, at least in the studied range of variations.

\section{Effects of the pore diameter (a)}

Once the perforation diameter and the pore arrangement are established, the pore diameter can be studied with the
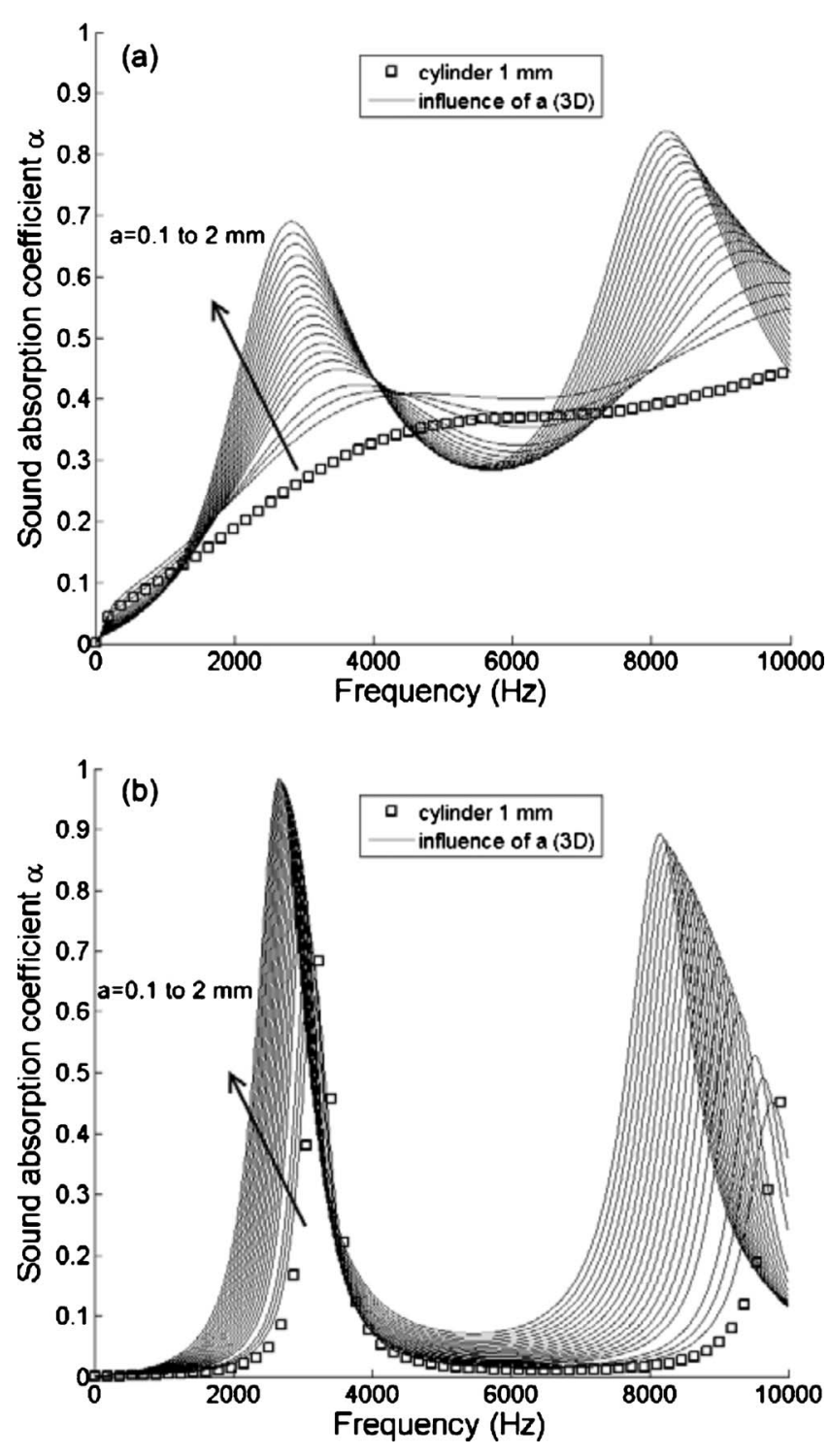

FIG. 10. Influence of the pore diameter on the normal sound absorption coefficient. (a) Maximum porosity case. (b) Intermediate porosity case.

2D-model. The 3D analytical correction can then be used to estimate the influence of this parameter on the 3D model. Here, the pore diameter $a$ varies between $D_{\text {hole }} / 10$ and $2 D_{\text {hole }}$. In the following simulations, the lined-up homogeneous configuration is used with $D_{\text {hole }}=1 \mathrm{~mm}, d=1.5 a, w$ $=0 \mathrm{~mm}$, and $L_{s}=25 \mathrm{~mm}$.

Figure 10 shows the 3D results obtained. In Fig. 10(a), a maximum porosity is considered, which means that elementary cells are side by side. This fixes the perforation density. The results of the calculation are compared to the case of the single cylindrical tube (i.e., $\phi=1$ ). In Fig. 10(b), an intermediate porosity is fixed by considering a perforation density of $n=30279$ perforations $/ \mathrm{m}^{2}$, corresponding to 20 elementary cells on a $29-\mathrm{mm}$ diameter sample. The results are compared to the case of a perforated solid of the same perforation density and diameter. For this latter case, $\phi=0.024$.

A first analysis of these figures shows that the pore size controls the selectivity of the normal sound absorption. The calculations showed that large pores increase the tortuosity, which explains why the maximum peak is shifted toward 
lower frequencies as the pore size increases. Naturally, a structure with small pores tends to behave like the reference cylindrical case.

Considering the maximum porosity case of Fig. 10(a), Eq. (26) predicts a specific perforation diameter of $D_{\text {spe }}^{c y l}$ $=0.15 \mathrm{~mm}$ for the cylinder and therefore $D_{\text {hole }} \cong 6.6 D_{\text {spe }}^{c y l}$. Consequently, for the smallest pore diameter case ( $a$ $=0.1 \mathrm{~mm}$ ), the absorption curve of the perforated foam is comparable to the $5 D_{\text {spe }}$ absorption curve of the perforated solid, see Fig. 7 at $\phi_{\max }$. When the pore diameter $a$ increases, the perforation density and the surface porosity decrease. Referring to Fig. 7, a reduction of the porosity makes the sound absorption increases and be more selective. This trend is also observed in Fig. 10(a) on the perforated foam as diameter $a$ increases.

Now, considering the intermediate porosity case of Fig. $10(\mathrm{~b})$, the predicted specific perforation diameter of the corresponding perforated solid is $D_{\text {spe }}^{\text {solid }}=3.6 \mathrm{~mm}$ and, using Eqs. (3) and (4), its resistance is $\sigma L_{s}=609 \mathrm{~Pa} \cdot \mathrm{s} / \mathrm{m}$. This time, the absorption curve of the perforated foam with the smallest pores $(a=0.1 \mathrm{~mm})$ is comparable to the $D_{\text {spe }} / 5$ curve of the perforated solid at $\phi=0.024$ in Fig. 7. For this case, the resistance is rather large compared to the specific resistance. This limits the absorption amplitude at the first peak. To improve the first peak sound absorption of this perforated solid, one needs to reduce its resistance $\sigma L_{s}$ or increase $D_{\text {hole }}$. For the perforated foam with the same perforations, Fig. 10(b) shows that the absorption can also be improved by increasing the pore diameter. In fact, increasing the pore diameter will decrease $\sigma L_{s}$ or apparently increase $D_{\text {hole. }}$.

In conclusion, the previous analysis has shown that the results found for the perforated solids can also be used to investigate the sound absorption properties of perforated closed-cell foams. However, the effects of the local parameters $(w, d$, and $a$ ) have to be taken into account, at least the pore diameter as a first approximation.

\section{Comparison with measurements}

The model does not aim at exactly quantifying the normal sound absorption coefficient of a perforated closed-cell metallic foam, but an experimental comparison would enable to confirm the validity of the previous trends by linking the above microstructure parameters to sound absorption properties. The real material is the closed-cell aluminum foam shown in Fig. 1. The sample has a $29-\mathrm{mm}$ diameter and a 26.4-mm thickness. Fifteen perforations have been drilled through the total thickness; the perforation diameter is $D_{\text {hole }}=1 \mathrm{~mm}$. The mean pore size, measured using an optical measuring microscope, is $a=3.62 \pm 0.57 \mathrm{~mm}$. The sample was tested in a Bruel \& Kjaer 4026 small impedance tube over the frequency range 500-6000 Hz. The sound absorption coefficient is negligible for the entire frequency range before drilling.

Two simulations have been achieved. The first is realized using the 2D model and the 2D/3D conversion with pore diameter, perforation diameter, and number of perforations as input local geometry parameters. The lined-up con-
TABLE II. Computed macroscopic parameters of the perforated aluminum foam sample.

\begin{tabular}{|c|c|c|c|c|}
\hline$\phi$ & $\begin{array}{c}\sigma \\
\left(\mathrm{Pa} \mathrm{s} \mathrm{m}^{-2}\right)\end{array}$ & $\alpha_{\infty}$ & $\begin{array}{c}\Lambda \\
(\mathrm{m})\end{array}$ & $\begin{array}{l}\Lambda^{\prime} \\
(\mathrm{m})\end{array}$ \\
\hline 0.283 & 8433 & 5.54 & $0.281 \times 10^{-3}$ & $1.504 \times 10^{-3}$ \\
\hline
\end{tabular}

figuration and the homogeneous pore distribution are considered with $d=1.5 a$ and $w=0 \mathrm{~mm}$. The simulation results obtained for the macroscopic parameters of the perforated foam are summarized in Table II. Second, a 2-mm thick perforated plate is considered at the front face of the sample to account for the densified skin created during foaming, see Fig. 1. In this case, an added mass correction has to be taken into account. ${ }^{11}$ For highly resistive backing materials, this correction is calculated as a dynamic equivalent tortuosity according to the method proposed by Atalla and Sgard. ${ }^{27}$

The simulations and the measurements are compared in Fig. 11. The main observation is that the measured sound absorption (dotted line) has a main peak at $975 \mathrm{~Hz}$ which reaches about $100 \%$ absorption, and a second peak strongly damped between 3000 and $4000 \mathrm{~Hz}$. The main absorption peak of the simulation without the perforated facing plate (solid line) is $400 \mathrm{~Hz}$ higher than the measured one, and its second peak reaches about $85 \%$. Now, considering the facing plate in the simulation (dashed line), the frequency position of the first peak fits with measurements, and the second peak is strongly attenuated, like for measurements. The correlation is not perfect but the model is able to represent the main features of the experimental results despite all approximations- it is able to capture the prevailing dissipation phenomena.

Also, Fig. 11 shows the sound absorption of a 26.4-mm thick solid sample with 15 perforations. Equations (3), (4), and (28) yields the following properties for this sample: $\phi$ $=0.018, \sigma L_{s}=858 \mathrm{~Pa} \cdot \mathrm{s} / \mathrm{m}$, and $D_{\text {spe }}^{\text {solid }} \cong 5 D_{\text {hole }}$. Its sound absorption is comparable to the $D_{\text {spe }} / 5$ curve in the first graph of Fig. 7. Its large resistance limits the amplitude of the sound absorption at the first peak. For the same perfora-

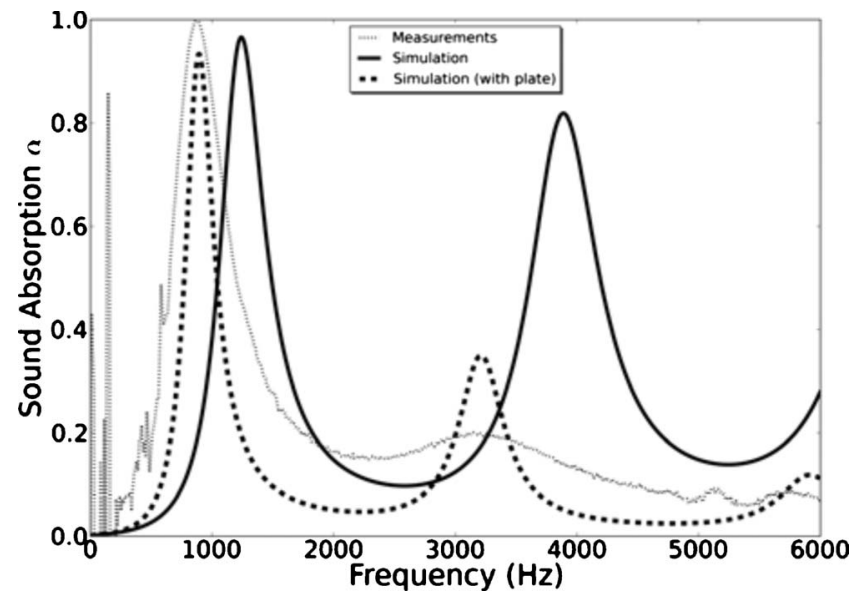

FIG. 11. Normal incidence sound absorption coefficient of a real perforated closed-cell aluminum foam. Measurements (dotted line) compared to numerical computations with (dashed line) and without (solid line) a perforated facing plate. 
tions, the perforated aluminum foam has a better sound absorption behavior. As previously discussed, this is due to the fact that its large pores have the effect to decrease $\sigma L_{s}$ toward the specific resistance compared to the perforated solid. Also, another divergence comes from the frequency location of the peaks between the cylinder and the real foam. The shift toward lower frequencies is due to the high tortuosity of the perforated foam as shown in Table II. In a first approximation, this tortuosity increases the effective thickness of the perforated foam by a factor of $\sqrt{\alpha_{\infty}}$. In Fig. 11, the maximum should then occur at $k_{0} L_{s} \sqrt{\alpha_{\infty}} \cong \pi / 2$, or at $f \cong 1376 \mathrm{~Hz}$, and not at $f \cong 3100 \mathrm{~Hz}$. This is in close agreement with the frequency at which the maximum occurs with the simulation without the facing plate.

\section{CONCLUDING REMARKS}

In this article, a microstructure based model was proposed to study the effects of perforations on the sound absorption of perforated closed-cell metallic foams. The theory of perforated solids was first revisited, then the given model was used to study the effects of the local geometry parameters on sound absorption of perforated closed cell metallic foams.

From the obtained results and comparisons with that for perforated solids, one can draw the main outcomes as follows: (1) For a perforated solid, a specific perforation diameter was identified and derived as a function of the solid sample thickness, and further given in terms of a power-law relation as a design guide. (2) From this analysis, a practical formula to predict the specific resistance was subsequently proposed. (3) It was shown that the perforated closed-cell metallic foam shows a similar behavior than a perforated rigid solid; however its sound absorption may be strongly modulated by its local geometry parameters, most particularly by the interaction between perforation diameter and pore size. (4) Finally, it was shown that the pore size may have a strong effect on the sound absorption frequency selectivity. This can be of advantage during the metallic foam manufacturing process if the noise spectrum to be tackled is known.

\section{ACKNOWLEDGMENT}

This work was supported in part by grants-in-aid from N.S.E.R.C. A part of the research presented in this paper was also supported by C.Q.R.D.A. and the Fond québécois de la recherche sur la nature et les technologies (F.Q.R.N.T.) by the intermediary of the Aluminum Research Centre-REGAL. C. Perrot acknowledges the partial financial support of this work by Université Paris-Est Marne-la-Vallée (Grant Number BQR-FG-354).

\footnotetext{
${ }^{1}$ M. F. Ashby, J. W. Hutchinson, and A. G. Evans, Cellular Metals, A Design Guide (Cambridge University Press, Cambridge, UK, 1998), Sec. 4.2 , pp. $42-47$
}

${ }^{2}$ T. J. Lu, A. Hess, and M. F. Ashby, "Sound absorption in metallic foams," J. Appl. Phys. 85, 7528-7539 (1999).

${ }^{3}$ T. Miyoshi, M. Itoh, S. Akiyama, and A. Kitahara, "Alporas aluminum foam: Production process, properties, and applications," Adv. Eng. Mater. 2, 179-183 (2000).

${ }^{4}$ T. J. Lu, F. Chen, and D. He, "Sound absorption of cellular metals with semiopen cells," J. Acoust. Soc. Am. 108, 1697-1709 (2000).

${ }^{5} \mathrm{M}$. A. Biot, "The theory of propagation waves in a fluid-saturated porous solid. I. Low frequency range," J. Acoust. Soc. Am. 28, 168-178 (1956).

${ }^{6} \mathrm{M}$. A. Biot, "The theory of propagation waves in a fluid-saturated porous solid. II. Higher frequency range," J. Acoust. Soc. Am. 28, 179-191 (1956).

'X. Wang and T. J. Lu, "Optimized acoustic properties of cellular solids," J. Acoust. Soc. Am. 106, 756-765 (1999).

${ }^{8}$ M.-Y. Zhou and P. Sheng, "First-principles calculations of dynamic permeability in porous media," Phys. Rev. B 39, 12027-12039 (1989).

${ }^{9}$ C. Perrot, F. Chevillotte, R. Panneton, and X. Olny, "Dynamic viscous permeability of an open-cell aluminum foam: Computations versus experiments," J. Appl. Phys. 103, p. 024909 (2008).

${ }^{10} \mathrm{C}$. Perrot, F. Chevillotte, and R. Panneton, "Bottom-up approach for microstructure optimization of sound absorbing materials," J. Acoust. Soc. Am. 124, 940-948 (2008).

${ }^{11}$ J. F. Allard and N. Atalla, Propagation of Sound in Porous Media. Modeling Sound Absorbing Materials, 2nd ed. (Wiley, Chichester, UK, 2009), Sec. 5, pp. 73-90, Sec. 9, pp. 188-198.

${ }^{12}$ D. L. Johnson, J. Koplik, and R. Dashen, "Theory of dynamic permeability and tortuosity in fluid-saturated porous media," J. Fluid Mech. 176, 379-402 (1987).

${ }^{13}$ Y. Champoux and J. F. Allard, "Dynamic tortuosity and bulk modulus in air-saturated porous media," J. Appl. Phys. 70, 1975-1979 (1991).

${ }^{14}$ S. R. Pride, F. D. Morgan, and A. F. Gangi, "Drag forces of porous media acoustics,” Phys. Rev. B 47, 4964-4978 (1993).

${ }^{15}$ D. Lafarge, P. Lemarinier, J. F. Allard, and V. Tarnow, "Dynamic compressibility of air in porous structures at audible frequencies," J. Acoust. Soc. Am. 102, 1995-2006 (1997).

${ }^{16} \mathrm{D}$. Lafarge, "Propagation du son dans les matériaux poreux à structure rigide saturés par un fluide viscothermique (Sound propagation in porous media saturated by a viscothermal fluid)," Ph.D. thesis, Université du Maine, France (1993).

${ }^{17}$ L. D. Landau and E. Lifshitz, Fluid Mechanics, 2nd ed. (ButterworthHeinemann, Oxford, 1987), Sec. 20, p. 58.

${ }^{18}$ R. J. S. Brown, "Connection between formation factor for electricalresistivity and fluid-solid coupling factor in Biot equations for acoustic waves in fluid-filled porous media," Geophysics 45, 1269-1275 (1980).

${ }^{19}$ Y. Champoux, "Étude expérimentale du comportement acoustique des matériaux poreux à structure rigide (Experimental study of the acoustic behavior of rigid porous materials)," Ph.D. thesis, Université de Sherbrooke, Carleton University, Canada (1991).

${ }^{20}$ Y. Champoux and M. R. Stinson, "On acoustical models for sound propagation in rigid frame porous materials and the influence of shape factors," J. Acoust. Soc. Am. 92, 1120-1131 (1992).

${ }^{21}$ M. Hakamada, T. Kuromura, Y. Chen, H. Kusuda, and M. Mabuchi, "High sound absorption level of porous aluminum fabricated by spacer method," Appl. Phys. Lett. 88, p. 254106 (2006).

${ }^{22}$ N. Geebelen, L. Boeckx, G. Vermeir, W. Lauriks, J.-F. Allard, and O. Dazel, "Measurement of the rigidity coefficients of a melamine foam," Acta Acust. Acust. 93, 783-788 (2007).

${ }^{23} \mathrm{X}$. Olny and C. Boutin, "Acoustic wave propagation in double porosity media," J. Acoust. Soc. Am. 114, 73-89 (2003).

${ }^{24} \mathrm{~T}$. H. Melling, "The acoustic impedance of perforates at medium and high sound pressure levels," J. Sound Vib. 29, 1-65 (1973).

${ }^{25}$ For a given porosity $\phi$, whatever the value of $L_{s}$ is, the shape of the function $\alpha\left(k_{0} L_{s}\right)$ is preserved and thus may be regarded as universal.

${ }^{26}$ The random nature of the pore distribution tends to impose a zero pore/ perforation relative position (i.e., $\bar{w}=0 \mathrm{~mm}$ ) in average.

${ }^{27} \mathrm{~N}$. Atalla and F. Sgard, "Modeling of perforated plates and screens using rigid frame porous models," J. Sound Vib. 303, 195-208 (2007). 